\title{
Erratum: Gauge and Yukawa coupling beta functions of two-Higgs-doublet models to three-loop order [Phys. Rev. D 97, 015016 (2018)]
}

Florian Herren, Luminita Mihaila, and Matthias Steinhauser

(Q) (Received 7 April 2020; published 20 April 2020)

DOI: 10.1103/PhysRevD.101.079903

In Eqs. (44) and (45), the coefficients of the last two terms had been interchanged in the preparation of the manuscript. They should read

$$
\beta_{1}=\ldots+\frac{\alpha_{1}^{2}}{(4 \pi)^{3}}\left[\ldots-\frac{6}{5} \hat{\lambda}_{i j, k l} \hat{\lambda}_{j i, l k}-\frac{3}{5} \hat{\lambda}_{i j, k l} \hat{\lambda}_{l i, j k}\right]
$$

and

$$
\beta_{2}=\ldots+\frac{\alpha_{2}^{2}}{(4 \pi)^{3}}\left[\ldots-2 \hat{\lambda}_{i j, k l} \hat{\lambda}_{j i, l k}-\hat{\lambda}_{i j, k l} \hat{\lambda}_{l i, j k}\right]
$$

Using Eq. (7) to rewrite the two combinations of quartic couplings in terms of the couplings given in Eq. (2) yields for the two structures in the above equations,

$$
\begin{aligned}
& \hat{\lambda}_{i j, k l} \hat{\lambda}_{j i l l k}=\lambda_{1}^{2}+\lambda_{2}^{2}+2 \lambda_{3}^{2}+2 \lambda_{4}^{2}+2\left|\lambda_{5}\right|^{2}+4\left|\lambda_{6}\right|^{2}+4\left|\lambda_{7}\right|^{2}, \\
& \hat{\lambda}_{i j, k l} \hat{\lambda}_{l i, j k}=\lambda_{1}^{2}+\lambda_{2}^{2}+4 \lambda_{3} \lambda_{4}+2\left|\lambda_{5}\right|^{2}+4\left|\lambda_{6}\right|^{2}+4\left|\lambda_{7}\right|^{2} .
\end{aligned}
$$

This implies that only terms containing $\lambda_{3}$ and $\lambda_{4}$ are affected by the change. The ancillary files [1] have been updated accordingly.

We thank Anders Eller Thomsen for pointing out the inconsistency with the three-loop gauge coupling beta function for a general quantum field theory [2] and our results.

[1] https://www.ttp.kit.edu/preprints/2017/ttp17-046/.

[2] C. Poole and A. E. Thomsen, J. High Energy Phys. 09 (2019) 055.

Published by the American Physical Society under the terms of the Creative Commons Attribution 4.0 International license. Further distribution of this work must maintain attribution to the author(s) and the published articles title, journal citation, and DOI. 